\title{
ASPECTOS CLÍNICOS DA HIPONATREMIA NA PRÁTICA ESPORTIVA
}

\author{
Clinical Aspects of Sports in Practice Hyponatremia
}

\author{
Anderson Martelli \\ Especialista em Laboratório Clínico / Patologia Clínica Faculdade Ciências Médicas / Unicamp \\ Docente Faculdade FMG / Mogi Guaçu \\ martellibio@hotmail.com
}

\section{RESUMO}

Vários distúrbios orgânicos no esporte são decorrentes de falhas nos esquemas de reposição hídrica e eletrolítica, que prejudicam sobremaneira a tolerância ao esforço e colocam em risco a saúde dos praticantes de exercícios físicos, podendo até mesmo causar a morte. Um dos distúrbios observados em modalidades esportivas de longa duração e de grande importância clínica são os quadros de hiponatremia, uma séria complicação orgânica de etiologia ainda desconhecida onde é observada uma baixa concentração de sódio plasmático. Assim, o objetivo deste trabalho é verificar através de uma revisão bibliográfica os mecanismos envolvidos nos quadros de hiponatremia na prática esportiva e as formas de prevenção deste importante quadro. Para a composição da presente revisão foi realizado um levantamento bibliográfico nas bases de dados Scielo, Lilacs, Portal de Periódicos da Coordenação de Aperfeiçoamento de Pessoal de Nível Superior (CAPES), a busca de dados no Google Acadêmico de artigos científicos e adicionalmente a consulta de livros acadêmicos para complementação das informações sobre os quadros de hiponatremia. Conclui-se que os distúrbios hidroeletrolíticos (hiponatremia e desidratação) são incidentes nas modalidades esportivas que demandam provas extensas, sendo a hiperhidratação a provável e principal etiologia deste distúrbio.

Palavras-chave: Hiponatremia; exercício; sódio; desidratação.

\begin{abstract}
Several organic disorders in sport are due to failures in water and electrolyte replacement schemes, which greatly impair exercise tolerance and pose physical exercise practitioner's health at risk and may even cause death. One of disturbances in sports long lasting and of great clinical importance are the frames of hyponatremia, a serious complication of organic unknown etiology in which a low concentration of plasma sodium is observed. The objective of this work is to verify through a literature review the mechanisms involved in cases of hyponatremia in sports and ways to prevent this important framework. For the composition of this review a literature in the Scielo, Lilacs, Journals Portal Coordination of Improvement of Higher Education Personnel (CAPES) data, data search in Google Scholar for papers and further consultation was conducted academic books to complement the information on the frames of hyponatremia. It is concluded that the electrolyte disturbances (hyponatremia and dehydration) are incidents in sports that require extensive testing, with overhydration being the likely main cause of this disorder.
\end{abstract}

Keywords: hyponatremia, exercise, sodium, dehydration

Persp. online: biol. \& saúde, Campos dos Goytacazes, 11 (3), 1-13, 2013

seer.perspectivasonline.com.br 


\section{INTRODUÇÃO}

A realização de atividades físicas seja com o objetivo competitivo, recreativo, terapêutico, militar ou laboral, deverá sempre estar associado a procedimentos que visem oferecer um maior conforto e segurança no campo da saúde corporal por parte dos praticantes de esportes, visando a manutenção do equilíbrio dos fluidos corporais e impedindo o aparecimento de um déficit hídrico que possa progredir para um quadro de desidratação (MARINS, 1998).

Sabe-se que a reposição hídrica adequada regula a circulação sanguínea, o volume plasmático e o controle da temperatura corporal. Não compensar a perda de líquidos, principalmente no exercício físico em ambientes quentes e úmidos, pode trazer sérias consequências ao atleta (PRADO et al., 2009).

Esse público excessivamente preocupado com a hidratação acaba utilizando a água como fonte principal de hidratação o que acarreta na potencialização da diluição do fluido extracelular e um aumento da quantidade de água corporal que os rins não conseguem compensar, isso, somado a uma baixa concentração de sódio corporal, ocasionam intoxicação por água ou hiponatremia (KASAPI, 2005; HIRATA et al., 2008).

Desde a primeira descrição da hiponatremia, as teorias expostas tentaram explicar sua etiopatogenia. Antes de 1981, o termo hiponatremia era totalmente desconhecido, sendo os atletas orientados a não ingerir líquidos durante os exercícios, o que poderia resultar em quadros de desidratação e hipernatremia. Após o ano de 1981, passou-se a recomendar para os atletas uma alta ingestão de líquidos, sem restrição durante exercícios de resistência aeróbica, entretanto, nesta época, houve um aumento dos casos de hiponatremia nos esportes de endurance e ultraendurance em consequência do recomendado (BORSATTO \& SPINELLI, 2007). Atualmente tem sido verificado que esta estratégia de reidratação de forma excessiva pode ser prejudicial à saúde dos atletas (MONTAIN et al., 1995).

Quando retratamos hidratação e reposição dos líquidos corporais, devemos levar em consideração que estes estão distribuídos no organismo nos compartimentos intra e extracelular, sendo esse último formado pelo interstício celular e plasma sanguíneo. Esses dois compartimentos, devido à permeabilidade seletiva da membrana endotelial possuem constituições semelhantes, mas concentrações distintas de solutos (Carvalho \& Mara, 2010).

Cerca de $20 \%$ do peso corporal são formados pelos líquidos intersticial e plasmático, respectivamente, $3 / 4$ e $1 / 4$ dos 14 litros do compartimento extracelular existentes em um homem de estatura média e pesando $70 \mathrm{~kg}$. Pesquisadores que atuam na área da medicina desportiva, 


\section{PERSPECTIVASonline}

classificam a hiponatremia como um desequilíbrio hidroeletrolítico, que resulta na queda anormal da concentração plasmática de sódio para valores abaixo de $135 \mathrm{mEq} / \mathrm{L}$ (KRATZ et al., 2005; NOAKES et al., 2005). Um estudo canadense envolvendo mais de 53.000 pacientes definiu hiponatremia como sódio $<138 \mathrm{mEq} / \mathrm{L}$ (Wald et al., 2010).

Em provas de longa duração, há uma importante perda hídrica através do suor e da respiração. Nestas condições, é preciso hidratar adequadamente de forma a não trazer riscos para a saúde do atleta, bem como para que não seja diminuído o seu rendimento físico no esporte praticado (BORSATTO \& SPINELLI, 2007).

Paradoxalmente, esportistas que ingerem importantes quantidades de água e que às vezes, excedem até mesmo as próprias necessidades, podem desencadear um aumento do volume plasmático, que leva a um funcionamento deficiente do aparelho excretor, impossibilitando o organismo de eliminar quantidades suficientes de água, resultando assim, em aumento do plasma sanguíneo e diluição do seu conteúdo dentre eles o íon sódio (WILMORE et al., 2010). Desta maneira, a hemodiluição resultante da hipervolemia pode resultar no quadro de hiponatremia também denominada de hiponatremia dilucional, ou seja, há mais água que o normal para a quantidade de substâncias dissolvidas no plasma. (PORCEL et al., 2004; ROCHA, 2011).

Segundo Carvalho e Mara (2010), na composição plasmática predominam os cátions de sódio (142 mEq1/L), secundados pelos ânions de cloro e pequenas quantidades de proteínas e potássio $(4,2 \mathrm{mEq} / \mathrm{L})$. A composição do líquido extracelular (LEC) é rigorosamente regulada por diversos mecanismos, com destaque para a função renal, o que mantém as células banhadas por um líquido com concentração de eletrólitos e nutrientes apropriada ao seu perfeito funcionamento.

Almond et al.(2005) observaram que durante a maratona de Boston de 2002, 13\% dos atletas apresentaram hiponatremia e três atletas apresentaram concentrações tão baixas de sódio plasmático que corriam risco de morte. Além disso, naquele estudo observou-se que muitos atletas excederam nas quantidades ingeridas de líquidos a ponto de ter sido verificado o aumento no seu peso corporal ao final do percurso da maratona.

O fato da hiponatremia ser fatal a atletas que não apresentam nenhum outro tipo de patologias é motivo suficiente para fazer com que os profissionais de saúde esportiva conheçam quais são os fatores de risco e como esse distúrbio pode ser evitado. Apesar da incidência de hiponatremia fatal ser rara, relatos de caso e dados descritivos sugerem que a hiponatremia não fatal é comum (MURRAY et al., 2003). Dados recentes têm demonstrado evidências sobre o crescente número de pessoas que são acometidas pela hiponatremia durante exercícios físicos prolongados, devido, sobretudo, à hiperidratação (SILVA et al., 2011).

Persp. online: biol. \& saúde, Campos dos Goytacazes, 11 (3), 1-13, 2013 
Sendo as alterações hidroeletrolíticas no esporte influenciadas principalmente pelas condições ambientais, condicionamento, hidratação e aclimatação do atleta, este artigo apresenta como objetivo verificar através de uma revisão bibliográfica a alteração hidroeletrolítica caracterizada como hiponatremia, quadro este encontrado em praticantes de esportes de longa duração, analisando o comportamento das concentrações sódio frente à hidratação excessiva, proporcionando um maior conhecimento sobre este desequilíbrio e os eventuais prejuízos aos atletas, comissão organizadora, e principalmente a equipe médica, visando uma abordagem preventiva, diagnóstica e terapêutica apropriada.

\section{DESENVOLVIMENTO}

Para a composição da presente revisão foi realizado um levantamento bibliográfico nas bases de dados Scielo, Lilacs, Portal de Periódicos da Coordenação de Aperfeiçoamento de Pessoal de Nível Superior (CAPES) e a busca de dados no Google Acadêmico de artigos científicos nacionais e internacionais publicados até 2011 utilizando como descritores isolados ou em combinação: hiponatremia e desequilíbrio hidroeletrolítico, exercícios físicos, concentração de sódio e desidratação e adicionalmente a consulta de livros acadêmicos para complementação das informações sobre os aspectos clínicos e os mecanismos da hiponatremia na prática esportiva.

Para seleção do material, efetuaram-se três etapas. A primeira foi caracterizada pela pesquisa do material. A segunda compreendeu a leitura dos títulos e resumos dos trabalhos, visando uma maior aproximação e conhecimento, sendo excluídos os que não tivessem relação e relevância com o tema. Após essa seleção, buscaram-se os textos que se encontravam disponíveis na íntegra, sendo estes, inclusos na revisão Figura 1.

Dentre os materiais de pesquisa, foram selecionados artigos escritos em português, inglês e espanhol, sendo a maioria deles escritos em língua portuguesa 18, inglês 12 e espanhol 1. Dos artigos selecionados e incluídos na pesquisa constituíram ensaios clínicos, artigos originais, revisões e revisões sistemáticas. Os critérios de elegibilidade foram estudos que apresentassem dados referentes aos quadros de hiponatremia desenvolvidos em provas esportivas de longa duração preconizados entre os anos de 1995 até o mais atual 2011. Na leitura e avaliação, os artigos que apresentaram os critérios de elegibilidade foram selecionados por consenso.

Persp. online: biol. \& saúde, Campos dos Goytacazes, 11 (3), 1-13, 2013 


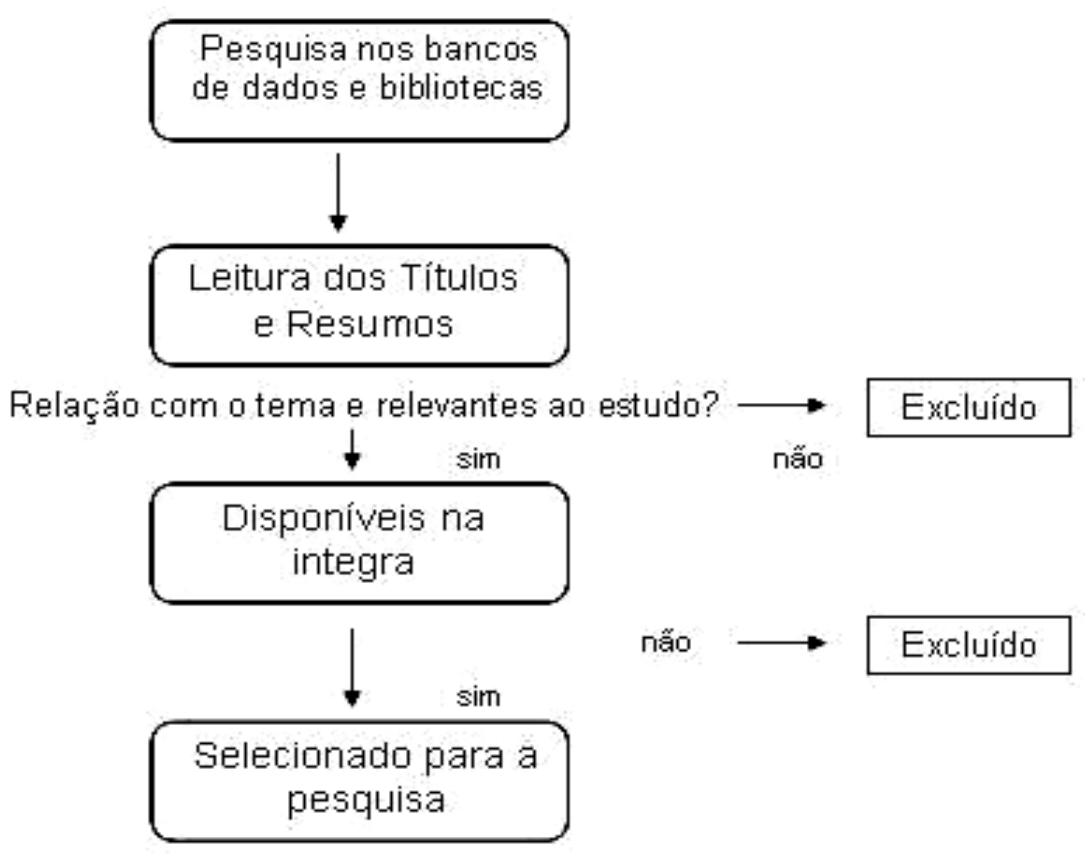

Figura 1. Fluxograma relativo às etapas de seleção dos artigos.

\section{HIPONATREMIA NO ESPORTE}

Os distúrbios hidroeletrolíticos nas provas esportivas de longa duração têm sido alvo de estudos por diversos pesquisadores. Neste contexto, os quadros de hiponatremia tem se apresentado de grande importância dentro da medicina do esporte, principalmente nos casos sintomáticos.

A hiponatremia é uma séria complicação orgânica de etiologia ainda desconhecida, que pode acometer particularmente os adeptos de esporte de forte resistência aeróbica e atletas de elite (SIMÕES et al., 2005; HIRATA et al., 2008). Dados recentes têm demonstrado evidências sobre o crescente número de pessoas que são acometidas pela hiponatremia (NOAKES, 2003).

Os quadros hiponatrêmicos que acometem os atletas, praticantes de provas longas é mais frequentemente conhecido como hiponatremia hipotônica ou dilucional, onde é observado uma quantidade de água acima do normal para a quantidade de substâncias dissolvidas no plasma. A hiponatremia isotônica (relação normal entre a água e as substâncias dissolvidas) é rara, mas pode ser a consequência da retenção de fluidos isotônicos que não contêm sódio no compartimento de fluidos extracelulares, o que é relevante para alguns procedimentos hospitalares como, por exemplo, as infusões isotônicas de manitol, mas não nos esportivos e por fim, a hiponatremia hipertônica, onde é observado um volume de água menor que o normal para a quantidade de substâncias dissolvidas no plasma, podendo ocorrer na presença de hiperglicemia grave ou de carga de glicerol 


\section{PERSPECTIVAS online}

quando a água retida no espaço vascular é suficiente para reduzir a concentração sanguínea de sódio temporariamente. (MURRAY et al., 2003)

Quando grandes quantidades de água corporal são perdidas, como durante o exercício de longa duração, o equilíbrio entre a água, os eletrólitos e o estoque de glicogênio pode ser rapidamente alterado e, a menos que esses elementos sejam repostos, podem ocorrer hipovolemia, hipoglicemia, hiponatremia, hipertermia e desidratação (DE BOCK et al., 2008).

Há muitas causas possíveis para explicar a hiponatremia relacionada ao exercício. Murray et al. (2003) descrevem que uma das hipóteses é a Síndrome da Secreção Inadequada de Hormônio Anti-Diurético (ADH) - a SIADH, onde ocorre uma redução na produção de urina e um aumento da retenção de fluidos ingeridos na presença de sobrecarga líquida. Uma segunda hipótese é a do sequestro da água no intestino, resultando em uma diluição após a competição, quando a água é absorvida e por fim, uma hipótese que está sendo relacionada ao uso abusivo de anti-inflamatórios não hormonais que podem alterar a função renal e reduzir a produção de urina.

Com a prática do exercício e durante a realização do mesmo, o atleta pode perder cerca de $240 \mathrm{mEq} . \mathrm{l}^{-1}$ de sódio, podendo progredir para um quadro de hiponatremia, ou seja, concentração de sódio plasmático menor que $135 \mathrm{mEq.1^{-1 }}$, ocasionado por uma reposição hídrica em excesso com líquidos que não possuem sódio ou concentração baixa, sendo observado a ocorrência deste quadro em eventos longos (HERNANDEZ \& NAHAS, 2009). Batista et al. (2007).

Rocha (2011) descreve que durante a sudorese, quando os eletrólitos são perdidos através do suor, os íons restantes são redistribuídos entre os tecidos corporais. Assim, na hiponatremia, o LEC se torna hipotônico em relação ao líquido intracelular (LIC), gerando desvio de água para o interior das células.

De acordo com Murray et al. (2003), os atletas que iniciam suas atividades com hiponatremia em função da ingestão excessiva de líquidos nos dias ou horas que antecedem a competição, correm um risco significativo de desenvolver um quadro grave deste distúrbio durante a competição porque menos fluido é necessário para fazer com que os níveis de sódio atinjam valores perigosos.

Segundo Siegel (2006), a gravidade dessa patologia classifica-se desde leve, com sódio = 131-134 mEq/L e geralmente sem sintomas, moderada, com sódio = 126-130 mEq/L podendo causar fraqueza, náuseas, fadiga, confusão e "phantom running" (movimento involuntário e persistente das pernas em descanso) até grave, com sódio < $126 \mathrm{mEq} / \mathrm{L}$ podendo causar coma, convulsões e até mesmo a morte figura 2.

Persp. online: biol. \& saúde, Campos dos Goytacazes, 11 (3), 1-13, 2013 
A manutenção desses baixos valores afeta o balanço osmótico na barreira hematoencefálica, causando a rápida entrada de água no cérebro, podendo progredir para um edema cerebral e uma cascata de respostas neurológicas cada vez mais grave, que podem culminar com a morte em consequência da lesão do tronco cerebral (HIRATA et al., 2008; ROCHA, 2011).

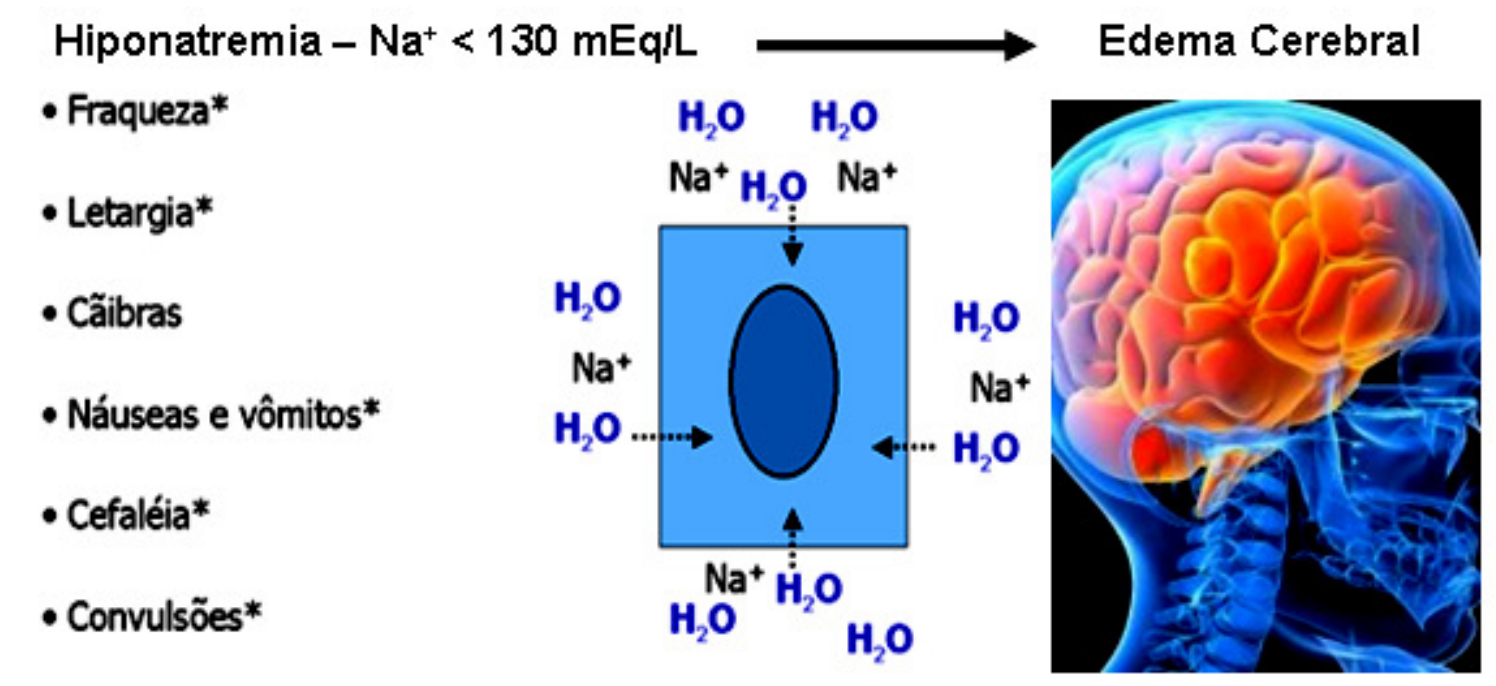

Figura 2. Hiponatremia moderada com $\mathrm{Na}^{+}<130 \mathrm{mEq} / \mathrm{L}$ e desequilíbrio osmótico com entrada de água no cérebro e progressão para edema cerebral. (*) Sintomas associado à entrada de $\mathrm{Na}^{+}$para dentro do neurônio.

De acordo com o estudo de Rocha (2011), a velocidade de instalação da hiponatremia é um fator determinante na sintomatologia, sendo observado que a instalação de um quadro de hiponatremia moderada aguda pode ser mais sintomática que uma severa crônica, isto porque, na hiponatremia crônica, mecanismos adaptativos entram em ação visando reduzir a osmolaridade intracelular e, consequentemente, minimizar a entrada de água nas células e uma progressão para edema cerebral. Para isso, os neurônios inicialmente excretam sais de sódio e potássio e, posteriormente, osmólitos orgânicos.

O esporte de alta performance requer um estudo detalhado sobre as influências fisiológicas durante sua prática (LIMA et al., 2007). Segundo Marin et al. (2003), o aumento da atividade muscular promove uma elevada produção de calor no organismo, que é eliminado em parte, pelo suor que no exercício de longa duração vai promover a perda de eletrólitos e de água no corpo, sendo o sódio, o principal componente eletrolítico deste meio.

Sendo hipotônico o suor em relação ao plasma, inicialmente a perda de água é proporcionalmente maior do que a de eletrólitos, em especial do sódio, ocorrendo desidratação com 
hipernatremia. Posteriormente, como se costuma oferecer mais água do que sódio pela ingestão de água pura ou de bebidas 'desportivas' com menor concentração de sódio do que a do plasma sanguíneo, o atleta pode progredir para um quadro de hiponatremia (CARVALHO \& MARA, 2010).

Indivíduos aclimatados apresentam menor perda de sal em relação aos não aclimatados, mas têm taxa maior de sudorese, podendo apresentar hiponatremia em atividades com mais de três horas de duração ao considerarmos o montante final de sudorese e a qualidade da reposição hídrica. (CARVALHO \& MARA, 2010).

Quando retratamos a reposição hídrica, a sede é resultante do aumento de concentração de sódio, incitando a pessoa a consumir mais água, que é então retida no compartimento extracelular (SILVA \& FERNADEZ et al., 2003).

Neste compartimento extracelular, a osmolaridade normal dos líquidos é restabelecida com o aumento do consumo de água, porem esse aumento os difunde, diluindo as outras substâncias presentes no meio (SILVA et al., 2011). O status da hidratação não é medido facilmente, mas as mudanças agudas no status da hidratação são estimadas frequentemente por alteração da massa corporal (MAUGHAN, 2007).

$\mathrm{Na}$ hiponatremia causada pela sobrecarga de líquidos, pode ocorrer uma diluição dos eletrólitos dentro da célula fazendo com que ela se inche (PEREIRA et al., 2004) e faz com que o atleta pareça inchado e anéis, relógio, sapatos e pulseiras de identificação para corrida costumam ficar apertadas (HIRATA et al., 2008). Entretanto, mesmo na ausência de outras causas, a ingestão excessiva de líquidos por si só pode resultar em hiponatremia, como acontece com aquelas pessoas que ingeriram grandes volumes de líquidos na tentativa de diluir a urina para evitar a detecção de substâncias proibidas durante os testes anti-doping (GARDNER, 2002).

Assim, a combinação causada pela perda de sódio e a ingestão excessiva de água é uma das principais causas do aparecimento da hiponatremia (MURRAY et al., 2003), levando até ao aumento de peso corporal pós competição. (MARINS et al., 2003). A hiperidratação com líquidos a base de glicerol pode facilitar a hiponatremia, pois favorece a diluição dos íons, e o atleta apresenta uma vontade maior de urinar durante a prática de exercício (HERNANDES \& NAHAS, 2009).

Neste sentido, o rim é considerado o órgão de extrema importância na manutenção da homeostase do corpo humano, além de eliminar produtos de degradação do sangue e regular os níveis de água, também está diretamente envolvido na homeostase do conteúdo de eletrólitos do organismo (SILVA et al., 2011).

Persp. online: biol. \& saúde, Campos dos Goytacazes, 11 (3), 1-13, 2013 
Alguns estudos realizados em situações de exercícios competitivos e de laboratório, relataram diminuições de 20 a $60 \%$ na função renal comprovando o que já é sabido sobre a alteração que a função renal pode sofrer durante o exercício, com consequente aumento na concentração da urina (ALMOND et al., 2005). Essa diminuição da função renal esta diretamente relacionada a diminuição do fluxo sanguíneo nos rins (ZAMBRANSKI, 1996).

Neste sentido, uma das possíveis explicações para a hiponatremia seria que, conforme uma pessoa durante o exercício consuma excessivamente líquidos, somada à função renal alterada, poderia ocasionar respostas fisiológicas negativas como: hemodiluição e deslocamento do excesso de água para o espaço intracelular, que pode ser fatal (MURRAY, 2003; MOREIRA et al., 2006).

A hiponatremia é incomum em provas com duração inferior a 4 horas e mais frequentes em provas com permanência acima de 8 horas (CORRÊA, 2005). Em exercícios de longa duração e com reposição de muitos líquidos com baixa concentração de sódio, percebe-se a ocorrência de hiponatremia, que associada à desidratação durante uma prova, compreende a necessidade de atendimento médico para os atletas, e em geral, após a rehidratação oral os sintomas desaparecem (MAUGHAN \& BURKE 2004).

Davis et al. (2001) relataram 26 casos de hiponatremia sintomática nas Maratonas de San Diego de 1998 e 1999. O tempo médio dos 26 atletas para terminar a prova foi de 5 horas e 38 minutos (variação de 4h a 6h34) e muitos admitiram beber o máximo possível de líquidos durante e após a maratona e a concentração plasmática de sódio variou de 117 a $134 \mathrm{mmol} / \mathrm{L}$, portanto a ingestão excessiva representa uma possibilidade bem convincente desta patologia.

Quanto a suplementação de bebidas com sódio para reposição, Maughan e Burke (2004) relatam esta necessidade quando houver grandes perdas líquidas através da transpiração e quando um volume grande de fluidos é consumido.

Para Machado-Moreira et al. (2010), a ingestão de acordo com a sede seria suficiente para garantir a reposição hídrica durante o exercício físico, pois o sistema nervoso central é tido como capaz de indicar corretamente o volume de fluido a ser ingerido, de acordo com as informações enviadas pelo sistema nervoso sobre a necessidade do organismo que garantam a regulação dos níveis plasmáticos e da temperatura do corpo.

Carvalho e Mara (2010) descrevem que em eventos de mais de três horas de duração, como ultramaratonas e triatlo Ironman, a intensidade de esforço situa-se entre 30 e $70 \%$ do consumo máximo de oxigênio e, além da reposição hídrica e do substrato energético, há necessidade do fornecimento de eletrólitos ao atleta, principalmente o sódio. Por exemplo, no final de uma prova de três horas de duração, com a taxa de sudorese de dois litros por hora, a ingestão de metade das 
perdas na forma de água pode resultar em hiponatremia, com níveis de sódio abaixo de 132mEq1/L no plasma.

Um ponto relevante que deve ser descrito é a relação massa corporal e hiponatremia. Apesar de atletas com porte físico maior não estarem imunes à hiponatremia, atletas menores e de provas lentas que suam muito, excretam um suor salgado e que são excessivamente cuidadosos com os hábitos relacionados à ingestão de líquidos são aqueles que correm um maior risco. Um porte físico menor significa que é preciso menos fluido para diluir o LEC (MURRAY et al., 2003).

Atualmente tem se falado muito na alimentação e hidratação de atletas. Uma ingesta em quantidades corretas de sódio pode reduzir o aparecimento da hiponatremia. $\mathrm{O}$ fato de a hiponatremia poder ser fatal a atletas que não apresentam nenhuma patologia é motivo suficiente para fazer com que os profissionais de saúde que lidam com o esporte conheçam quais são os fatores de risco e como esse distúrbio pode ser evitado (INÁCIO et al., 2010).

\section{CONCLUSÃO}

Dentre os distúrbios eletrolíticos observados em modalidades esportivas de longa duração e de grande importância clínica, descrevemos os quadros de hiponatremia, uma séria complicação orgânica de etiologia ainda desconhecida onde é observada uma baixa concentração de sódio plasmático e/ou diluição deste devido a hiperhidratação.

Nessas modalidades esportivas, o atleta deve ser orientado não somente quanto aos riscos da desidratação, mas também, quanto à hiperrhidratação ou sobrecarga de fluidos, a qual podem desencadear complicações como a hiponatremia sintomática.

Protocolos de prevenção, diagnóstico e terapêutica devem ser sistematicamente instituídos visando a redução da incidência dos distúrbios hidroeletrolíticos em atletas praticantes de eventos de longa duração e em paralelo maiores estudos científicos sobre os quadros de hiponatremia, visando consolidar dados sobre a prevenção, visto o alto grau de periculosidade no meio esportivo, principalmente porque a hiponatremia pode levar a morte de atletas.

As discussões e os estudos ainda continuam para que deixem clara a real causa da hiponatremia induzida pelo exercício intenso. Assim, é de extrema importância que membros de comissões técnicas, dirigentes do esporte e demais profissionais do meio esportivo, dentre eles o profissional de Educação Física, se atentem para a prevenção da hiponatremia, e também para identificar possíveis sintomas visando uma intervenção antecipada.

Persp. online: biol. \& saúde, Campos dos Goytacazes, 11 (3), 1-13, 2013

seer.perspectivasonline.com.br 


\section{REFERENCIAS BIBLIOGRAFICAS}

ALMOND, C. S.; SHIN, A. Y.; FORTESCUE, E. B.; MANNIX, R. C.; WYPIJ, D.; BINSTADT, B. A.; et al. Hyponatremia among runners in the Boston Marathon. N Engl J Med. n. 352, p. 1550$56,2005$.

BATISTA, M. A. L.; FERNANDES FILHO, J.; DANTAS, P. M. S. A influência da intensidade de treinamento e a perda de peso no futebol. Fit Perform J. n. 6, p. 251-25, 2007.

BORSATTO, J. E.; SPINELLI, N. C. Atletas de endurance e ultraendurance - uma investigação sobre efeitos da hiponatremia. Revista de Educação Física. n. 139, 2007.

CARVALHO, T.; MARA, L. S. Hidratação e Nutrição no Esporte. Rev Bras Med Esporte. v. 16, n. 2, Mar/Abr, 2010.

CORRÊA, E. C. M. Triatlo. In: BIESEK S, ALVES L. A, GUERRA I. Estratégias de nutrição e suplementação no esporte. $1^{\text {a }}$ ed. São Paulo: Ed Manole, 2005:434 - 448.

DAVIS, D.P.; VIDEEN, J.S.; MARINO, A. et al. Exercise-associated hyponatremia in marathon runners: a two-year experience. J Emerg. Med. v. 21, p.47-57, 2001.

DE BOCK, K.; DERAVE, W.; EIJNDE. et al. Effect of training in the fasted state on metabolic responses during exercise with carbohydrate intake. J Appl Physiol. n. 104, p. 1045-55, 2008.

GARDNER, J.W. Fatal water intoxication of an Army trainee during urine drug testing. Military Med. v. 5, p.435-437, 2002.

HERNANDEZ, A. J.; NAHAS, R. M. Modificações dietéticas, reposição hídrica, suplementos alimentares e drogas: comprovação de ação ergogênica e potenciais riscos para a saúde. Rev. Brás. Méd. Esporte. v. 15, n. 3, mai/jun, p. 3 - 12, 2009.

HIRATA, N. R.; VIST, P.; LIBERALI, R. Hiponatremia em Atletas. Revista Brasileira de Nutrição Esportiva, São Paulo v. 2, n. 12, p. 462-471, Novembro/Dezembro, 2008.

INÁCIO, G. S.; COSTA, E. M.; AGUIAR, N. B. C.; CUNHA, R. M. Hiponatremia e o esporte. EFDeportes.com, Revista Digital. Buenos Aires, Año 15, n. 149, 2010.

KAPASI, I.A.M.; ALBIERO, K.A.; STÜRNER, K. Aspectos para Minimizar os Efeitos Adversos da Prática Esportiva no Calor. Rev. Nut. em Pauta, n.72, maio/jun., 2005.

KRATZ, A.; SIEGEL, A. J.; VERBALIS, J. G. et al. Sodium status of collapse marathon runners. Arch Pathol Lab Med. n. 129, p. 227-30, 2005.

LIMA, C.; MICHELS, M.F.; AMORIM, R. Os diferentes tipos de substratos utilizados na hidratação do atleta para melhora no desempenho. Revista Brasileira de Nutrição Esportiva, v. 1, n. 1, p. 73-83, jan/fev, 2007.

MAUGHAN, R. J; BURKE, L. M. Nutrição Esportiva. 1ª ed. Porto Alegre: Ed. Artmed, 2004.

Persp. online: biol. \& saúde, Campos dos Goytacazes, 11 (3), 1-13, 2013

seer.perspectivasonline.com.br 
MARINS, J. C. B.; DANTAS, E. H. M.; NAVARRO, S. Z. Diferentes tipos de hidratação durante o exercício prolongado e sua influência sobre o sódio plasmático. Rev. Brás. Ciên. e Mov. v. 11, n. 1, p. $13-22$, jan. 2003.

NOAKES, T. D.; SHARWOOD, K.; SPEEDY, D. et al. Three independent biological mechanisms cause exercise-associated hyponatremia: evidence from 2,135 weighed competitive athletic performances. PNAS. v. 102, n. 51, p. 18550-5, 2005.

MACHADO-MOREIRA, C.A.; VIMIEIRO-GOMES, A.C.; SILAMI-GARCIA, E; RODRIGUES, L.C. Hidratação durante o exercício: a sede é suficiente? Rev Bras Med Esporte, v.12, n.6, Nov/Dez, 2006.

MARINS, J. C. B. Homeostase hídrica corporal em condições de repouso e durante o exercício físico. Revista Brasileira de Atividade Física \& Saúde, v. 3, n.2, p. 58-72, 1998.

MAUGHAN, R. J.; SUSAN, S. M.; LEIPER, J. B. Errors in the estimation oh hydration status from changes in body mass. J. of Sports Sci. Reino Unido, v. 25, p. 797-804, 2007.

MONTAIN, S. J.; LATZKA, W. A.; SAWKA, M. N. Control of thermoregulatory sweating is altered by hydration level and exercise intensity. J Appl Physiol. n. 79, p. 1434-39, 1995.

MOREIRA, M. C. A.; GOMES, A. C. V.; GARCIA, E. S.; RODRIGUES, L. O. C. Hidratação durante o exercício: a sede é suficiente? Rev Bras Med Esporte. n. 12, p. 405-09, 2006.

MURRAY, B.; EICHNER, E. R. E.; STOFAN, J. Hiponatremia em atletas. Sports Science Exchange. v. 16, n. 1, abr/mai/jun, 2003.

NOAKES, T. D. Overconsumption of fluids by athletes. BMJ, n. 327, p. 113-114, 2003.

PEREIRA, L. O.; MARQUEZI, M. L.; LACHA JUNIOR, A. H. Reposição Hídrica In: LANCHA JUNIOR, A. H. Nutrição e metabolismo aplicados à atividade motora. $1^{\text {a }}$ ed. São Paulo: Editora Atheneu, p. 95 - 130, 2004.

PORCEL, F. B. O.; RUIZ, J. R.; GARZÓN, M. J. C.; et al. Hiponatremia en esfuerzos de ultraresistencia: efectos sobre la salud y el rendimento. Archivos Latino Americanos de Nutricion. v. 54, n. 2, p. 155-64, 2004.

PRADO, E. S.; BARROSO, S. S.; GÓIS, H. O.; REINERT, T. Estado de hidratação em nadadores após três diferentes formas de reposição hídrica na cidade de Aracaju - SE. Fit Perf J. v. 8, n. 3, p. 218-25, 2009.

ROCHA, P.N. Hiponatremia: conceitos básicos e abordagem prática. J Bras Nefrol. v.33, n. 2, p.248-260, 2011.

SIEGEL, A. J. Exercise-Associated Hyponatremia: Role of Cytokines. Amer. Journal Med., USA, v. 119, n. 7A, p. 74-78, july, 2006.

SILVA, A. I.; FERNANDEZ, R. Dehydration of football referees during a match. J Sports Med. 2003;37:502-06. 
SILVA, F. I. C.; SANTOS, A. M. L.; ADRIANO, L. S.; LOPES, R. S.; VITALINO R, S. A. A importância da hidratação hidroeletrolítica no esporte. Rev. Bras. Cien. e Mov. v. 19, n. 3, p. 120$128,2011$.

SIMÕES, N. V. N. Lesões desportivas em praticantes de atividade física: uma revisão bibliográfica. Rev. Bras. Fisioter. v. 9, n. 2, p. 123-128, 2005.

WALD, R.; JABER, B.L.; PRICE, L.L. Upadhyay A, Madias NE. Impact of hospital-associated hyponatremia on selected outcomes. Arch Intern Med. v.170, p. 294-302, 2010.

WILMORE, J. H.: COSTILL, D. L.; KENNEY, W.L. Fisiologia do esporte e do exercício. Barueri: Manole, 2013.

ZAMBRASKI, E. J. The kidney and body fluid balance during exercise. In: Burskirk, E.R., Puhl, S.M. Body fluid balance: exercise and sport. Boca Raton: CRC, 1996. 